\title{
Numerical Solution of a Three-Phase Stefan Problem with High Power Input
}

\author{
Guido Parissenti ${ }^{1,1}$, Alfonso Niro ${ }^{1,2}$, \\ ${ }^{a}$ Politecnico di Milano, Dipartimento di Energia \\ Via Lambruschini 4, 20156 Milano - Italy
}

\begin{abstract}
The numerical solution of a one dimensional, three-phases Stefan problem with a low Stefan number is presented. Joule heating and thermal radiation are demonstrated to be negligible compared to the high power input. The Front Tracking Method is used along with a $2^{\text {nd }}$ order Lagrangian interpolation of the temperature profile near the moving surface defined by the location of the phase change. Results are compared with analytical, numerical and experimental solutions available in literature.
\end{abstract}

Keywords: $\quad$ electrode spot, phase change, Stephan problem

\footnotetext{
${ }^{1}$ guido.parissenti@mail.polimi.it (corresponding author)

2alfonso.niro@polimi.it
} 


\section{Introduction}

Being vacuum the best dielectric it is obvious its application in technological situations involving high current levels to be shut off safely and as instantaneously as possible. Vacuum switches apply this principle and for this reason they have a key role in many high power electrical systems. One of the problems affecting such devices is the appearance of electric arcs between the electrodes due to the sometimes extremely high current level involved. Arcs happen because absolute vacuum is not achievable and few electric carriers are always present. The intense heat fluxes of arcs can damage switches by melting the electrodes locally. In fact these phenomena happen in small zones that appear like small spots after the arc. These spots, with different shapes and dimensions, can be found on either electrodes causing erosion. Because spots appear to be a key element in understanding arc formation and extinction, a Stefan multiphase thermal model of the spot formation is here presented. Historically, for this kind of problem very few analytical solutions have been found and only for the simplest cases with no more than two phases [1][2], applied for infinite or semi-infinite regions. The same solution can instead be easily obtained numerically [3] with the possibility of solving more difficult problems [4]. Three phase problems have been already solved numerically in the past [5] but using complex and rigid finite-element methods, unsuitable to be inserted into larger codes for the simulation of complex physical problems involving not exclusively thermal phenomena. Hence the

purpose of this paper is to describe the application of a common finite dif- 
ference technique coupled with the front tracking method to this complex multiphase problem. We will show the flexibility of the formulation as well as that the results obtained are in good agreement with the literature. Due to its simplicity and low computation time, the presented approach can be used to preliminary evaluate damages occurring on electrodes surface during initial sizing and design of vacuum switches. In addition the presented method can be applied to every application which involves concentrated high power deposition on surfaces, i.e. electric thrusters for in-space propulsion or even laser ablation. More complex and multidimensional methods can be successively applied to increase the detail of the solution [6][7][8]. 


\section{Physical description of the thermal model}

Spots are small craters formed by an electric arc onto the electrode surface and they are usually round with a melted internal surface. The image in Figure 1 shows some spots cratered by an arc with a current density on the order of $10^{9} \mathrm{~A} / \mathrm{m}^{2}$ on an Aluminum anode.

[Figure 1 about here.]

Single spots from high-current electric arcs usually are much smaller than the electrode where they form $[9,10,11,12]$. In addition, the depth of the spot is much smaller, at least three times, than its diameter [9, 12]. From an analytical point of view, Lehr et al. [13] show that if the diffusion length $\left(\alpha_{s} t_{d}\right)^{1 / 2}$ is four times smaller than the electrode thickness by the incident heat flux direction, the electrode itself can be considered infinite in extent $\left(t_{d}\right.$ is a phenomenon characteristic time like the total discharge time, e.g. near $1 \mathrm{~ms}$ ). For copper this leads to a diffusion length of $0.35 \mathrm{~mm}$, much smaller than the thickness of a typical electrode. Therefore, the problem can be considered as one-dimensional. A schematic representation of the model is sketched in Figure 2.

The model can be described as a semi-infinite solid (approximate as a slab of length $\left.a \gg\left(\alpha_{s} t_{d}\right)^{1 / 2}\right)$ in the region $X>0$, where $X$ is the spatial coordinate perpendicular to the surface, where the heat flux $F(t)$ is applied at $X=0$. Additional terms for radiation as well as for Joule heating are else considered. The $F(t)$ flux first raises the solid temperature to the melting value $T_{m}$ 
(Stage 1 ). At this time $t_{1}$ a new phase appears with a new domain for the liquid phase. Hence, the original slab is split into two different time-varying domains. While the surface $S_{1}$ moving following Eq. (3), the temperature in the liquid phase starts rising until the vaporization temperature $T_{v}$ is attained at $t=t_{2}$ (Stage 2). At this time, the surface $S_{2}$ appears and starts moving following a relation similar to those for $S_{1}$ (Stage 3$)$.

We assume all thermal properties of the electrode are constant. Although in the vapor phase a temperature profile does not exist because vapor is assumed to be removed as soon as it forms, in the solid and liquid phases the temperature dynamic is ruled by the heat diffusion equations. At each moving surface, the Stefan Equation (described by Eq. 3,4) is introduced, to take into account the energy balance between the heat flux coming from the two phases and the change in internal energy due to the melting or vaporization. For the numerical solution, obviously, it is not possible to consider a semi-infinite domain, so we consider the solid as a slab in the domain $0<X<a$ with $a \gg r_{d}$. Because of this hypothesis, we assume that at $X=a$ temperature should always remain equal to the initial temperature $T_{i}$, and temperature gradient is null. The verification of these conditions means that the region remains unchanged, nevertheless only one of the two conditions (Dirichlet or Neumann - null temperature gradient) can be imposed: fixing one of these two boundary conditions at $X=a$ in the finite differences code is only a matter of choice as both should be verified after the simulation, to check whether $a$ has been taken sufficiently large. We decided 
to impose the Dirichlet condition, except in comparing to other codes, where the test case condition were used. Summarizing, the mathematical model can be described as follows.

[Figure 2 about here.]

Heat Diffusion Equations

$$
\begin{aligned}
k_{s} \frac{\partial^{2} T_{s}}{\partial X^{2}}+\eta_{s} j^{2} & =\rho c_{s} \frac{\partial T_{s}}{\partial t} \quad \text { for } \quad S_{1}<X<a \\
k_{l} \frac{\partial^{2} T_{l}}{\partial X^{2}}+\eta_{l} j^{2} & =\rho c_{l} \frac{\partial T_{l}}{\partial t} \quad \text { for } \quad S_{2}<X<S_{1}
\end{aligned}
$$

Stefan Equations on moving surfaces

$$
\begin{aligned}
k_{s} \frac{\partial T_{s}}{\partial X}-k_{l} \frac{\partial T_{l}}{\partial X} & =\rho C_{m} \frac{d S_{1}}{d t} \text { for } \quad X=S_{1}, t>t_{1} \\
k_{l} \frac{\partial T_{l}}{\partial X}+F(t) & =\rho C_{v} \frac{d S_{2}}{d t} \text { for } \quad X=S_{2}, t>t_{2}
\end{aligned}
$$


Boundary conditions

$$
\begin{aligned}
T_{s}=T_{i} \quad \text { for } \quad X=a \\
-k_{s} \frac{\partial T_{s}}{\partial X}=F(t) \quad \text { for } \quad X=0, t<t_{1} \\
-k_{l} \frac{\partial T_{l}}{\partial X}=F(t) \quad \text { for } \quad X=0, t_{1}<t<t_{2} \\
T_{s}=T_{l}=T_{m} \quad \text { for } \quad X=S_{1}, t>t_{1} \\
T_{l}=T_{v} \quad \text { for } \quad X=S_{2}, t>t_{2}
\end{aligned}
$$

Initial conditions

$$
\begin{aligned}
& T_{s}=T_{i} \text { for } t=0 \\
& S_{1}=0 \text { for } t<t_{1} \\
& S_{2}=0 \text { for } t<t_{2}
\end{aligned}
$$

Liquid and solid are considered to have the same density $\rho$ to allow a simpler manipulation of the Stefan equations and to avoid thermal expansion. The error is no larger than around $10 \%$ for both copper and aluminum.

\section{Finite Differences discretization and Front Tracking Method}

The most difficult issue in using a finite difference method for this kind of problem is the time-dependance of the domains. We cannot assume the moving boundaries always lie on a node of the mesh, and therefore we do not have a unique domain. Considering two separate domains with different 
discretizations is also difficult, because at the beginning of the simulation one of them will be very small or null. To bypass these problems is it possible to use the Front Tracking method [2]. The positions $S_{1}$ and $S_{2}$ of the moving boundaries are obtained from the integration of the Stefan equations (3) and (4), and therefore are not required to be a multiple of the mesh size. Let us say that at any time $k \delta t$ the phase-change boundary is located between two consecutive grid points, for example $i \delta X$ and $(i+1) \delta X$. To be able to define the finite difference form of the heat transfer equations (1) and (2) for these two points, we need to use a second order Lagrangian interpolation to allow unequal spatial intervals. We will see that the appearance of the solid-liquid boundary requires us to lower the order of the interpolation to one for the initial step because the domain is restricted to only one node.

As previously mentioned, this model considers heat generation and thermal radiation. The former is the term $\eta j^{2}$ in Eqs. (1) and (2) due to the current that flows into the spot and resistively heats the material. The thermal radiation can be considered in a very simple way by dividing $F(t)$ into two parts:

$$
F(t)=\tilde{F}(t)-q_{r}(t) \quad \text { where } \quad q_{r}(t)=\sigma \varepsilon\left(T_{\text {surf }}^{4}-T_{i}^{4}\right)
$$

where $q_{r}(t)$ is the heat flux that leaves the external surface (solid or liquid, depending on the phase status). $\tilde{F}(t)$ can be considered the external heat 
flux that flows into the anode, depending on the physics of the problem.

\section{Non-dimensionalization}

The first operation is the non-dimensionalization of the equations. The advantages to this operation are a simplification in writing the finite-difference form and the ability to obtain the Stephan number, a dimensionless value that gives information about the problem dynamics. Some characteristic parameters are chosen for this operation.

$$
\begin{aligned}
& x=\frac{X}{r} \quad x_{s, l}=\frac{X_{s, l}}{r} \quad s_{1,2}=\frac{S_{1,2}}{r} \\
& \tau=\frac{k_{s} t}{c_{s} \rho r^{2}} \\
& u_{s}=\frac{T_{s}}{T_{m}} \quad u_{l}=\frac{T_{l}}{T_{m}} \quad u_{v}=\frac{T_{v}}{T_{m}}
\end{aligned}
$$

All the symbols are explained R2 in the Nomenclature section.

Substituting these dimensionless variables into Eqs. (1) and (2) we have:

$$
\begin{gathered}
\frac{\partial^{2} u_{s}}{\partial x^{2}}+g_{s}=\frac{\partial u_{s}}{\partial \tau} \quad \text { for } \quad s_{1}<x<\frac{a}{r} \\
\frac{\partial^{2} u_{l}}{\partial x^{2}}+g_{l}=\tilde{k} \frac{\partial u_{l}}{\partial \tau} \quad \text { for } \quad s_{2}<x<s_{1}
\end{gathered}
$$

where

$$
\tilde{k}=\frac{k_{s}}{k_{l}} \frac{c_{l}}{c_{s}} \quad g_{s}=\frac{\eta_{s} j^{2} r^{2}}{k_{s} T_{m}} \quad g_{l}=\frac{\eta_{l} j^{2} r^{2}}{k_{l} T_{m}}
$$


Eq. (3) and (4) can be manipulated in the same way:

$$
\begin{gathered}
\gamma_{s} \frac{\partial u_{s}}{\partial x}-\gamma_{l} \frac{\partial u_{l}}{\partial x}=\frac{d s_{1}}{d \tau} \quad \text { for } \quad x=s_{1}, \tau>\tau_{1} \\
\gamma_{l v} \frac{\partial u_{l}}{\partial x}+f(\tau)=\frac{d s_{2}}{d \tau} \quad \text { for } \quad x=s_{2}, \tau>\tau_{2}
\end{gathered}
$$

where

$$
\begin{aligned}
& \gamma_{s}=\text { Ste } \quad \gamma_{l}=\frac{k_{l} \text { Ste }}{k_{s}} \quad \text { Ste }=\frac{c_{s} T_{m}}{C_{m}} \\
& \gamma_{l v}=\frac{k_{l} \lambda_{v}}{k_{s}} \quad f(\tau)=\frac{F(t) r \lambda_{v}}{k_{s} T_{m}} \quad \lambda_{v}=\frac{c_{s} T_{m}}{C_{v}}
\end{aligned}
$$

The Stefan number Ste expresses the importance of sensible heat relative to latent heat. For metals like aluminum, copper, tin, etc. the Stefan number is small, in the order of the unity. This means that the heat released or absorbed by the interface during phase change is mostly unaffected by the variation of internal thermal energy of the material [14]. The boundary and initial conditions can be transformed as follows: 


$$
\begin{gathered}
u_{s}=u_{i} \text { for } x=\frac{a}{r} \\
\frac{\partial u_{s}}{\partial x}=-f_{s}(\tau) \text { for } x=0, \tau<\tau_{1} \\
\frac{\partial u_{l}}{\partial x}=-f_{l}(\tau) \text { for } x=0, \tau_{1}<\tau<\tau_{2} \\
u_{s}=u_{l}=u_{B}=1 \text { for } x=s_{1} \\
u_{l}=u_{v} \text { for } x=s_{2} \\
u_{s}=u_{i} \text { for } \tau=0 \\
s_{1}=0 \text { for } \tau<\tau_{1} \\
s_{2}=0 \text { for } \tau<\tau_{2}
\end{gathered}
$$

where

$$
f_{s}(\tau)=\frac{F(t) r}{k_{s} T_{m}} \quad f_{l}(\tau)=\frac{F(t) r}{k_{l} T_{m}}
$$

\section{Lagrangian type interpolation}

The Lagrangian type interpolation method used by Crank [2] allows a modification of the finite difference formulae incorporating unequal spatial intervals near the moving boundary. Using a $2^{\text {nd }}$ order scheme based on three points, a generic function $u(x)$ can be represented as 


$$
\begin{gathered}
u(x)=\sum_{j=0}^{2} l_{j}(x) u\left(a_{j}\right) \\
l_{j}(x)=\frac{p_{2}(x)}{\left.\left(x-a_{j}\right) \frac{d p_{2}(x)}{d x}\right|_{x=a_{j}}} \quad p_{2}(x)=\left(x-a_{0}\right)\left(x-a_{1}\right)\left(x-a_{2}\right)
\end{gathered}
$$

Where $u\left(a_{0}\right), u\left(a_{1}\right), u\left(a_{2}\right)$ are three known values of $u(x)$ at the points $x=a_{0}, a_{1}, a_{2}$. From $u(x)$, is it possible to obtain the $1^{\text {st }}$ and $2^{\text {nd }}$ derivatives expressed in terms of $a_{j}$.

[Figure 3 about here.]

Referring to Fig. 3, where the moving boundary between liquid and solid is shown to be at a fractional distance $p d x$ from the considered node $i$, it is possible to specialize the above formulae to obtain the derivative in these two spatial intervals. Substituting values for $a_{j}$ and $u\left(a_{j}\right)$, pertaining to the solid phase

\begin{tabular}{c|c}
$\mathbf{a}_{\mathbf{j}}$ & $\mathbf{u}\left(\mathbf{a}_{\mathbf{j}}\right)$ \\
\hline$a_{0}=(i+1-(1-p)) d x=(i+p) d x$ & $u_{B}$ \\
$a_{1}=(i+1) d x$ & $u_{s}^{i+1}=u_{s}^{1}$ \\
$a_{2}=(i+2) d x$ & $u_{s}^{i+2}=u_{s}^{2}$
\end{tabular}

we write the derivatives

$$
\frac{\partial^{2} u_{s}}{\partial x^{2}}=\frac{1}{d x^{2}}\left(\frac{u_{B}}{(1-p)(2-p)}-\frac{u_{s}^{1}}{1-p}+\frac{u_{s}^{2}}{2-p}\right)
$$


for $x=(i+1) d x$, the first node of the solid domain, and

$$
\frac{\partial u_{s}}{\partial x}=\frac{1}{d x}\left(\frac{2-3 p}{(1-p)(2-p)} u_{B}+\frac{2-p}{1-p} u_{s}^{1}-\frac{1-p}{2-p} u_{s}^{2}\right)
$$

for $x=s_{1}(t)$ the interpolated point. The last derivative will be used for the Stefan equation. Analogously for the derivative of the liquid temperature profile we can substitute

$$
\begin{array}{c|c}
\mathbf{a}_{\mathbf{j}} & \mathbf{u}\left(\mathbf{a}_{\mathbf{j}}\right) \\
\hline a_{0}=(i-1) d x & u_{l}^{i-1}=u_{l}^{n_{l}-1} \\
a_{1}=i d x & u_{l}^{i}=u_{l}^{n_{l}} \\
a_{2}=(i+p) d x & u_{B}
\end{array}
$$

obtaining

$$
\frac{\partial^{2} u_{l}}{\partial x^{2}}=\frac{2}{d x^{2}}\left(\frac{u_{l}^{n_{l}-1}}{p+1}-\frac{u_{l}^{n_{l}}}{p}+\frac{u_{B}}{p(p+1)}\right)
$$

for $x=i d x$, the last node of the liquid domain, and

$$
\frac{\partial u_{l}}{\partial x}=\frac{1}{d x}\left(\frac{p}{p+1} u_{l}^{n_{l}-1}-\frac{(p+1)}{p} u_{l}^{n_{l}}+\frac{2 p+1}{p(p+1)} u_{B}\right)
$$

for $x=(i+p) d x$, the interpolated point. Eq. (28) should be used for the first node of the mesh, and (30) for the last node of the mesh. Eq. (29) and (31) are to be used in Eq. (16). These substitutions produce the finite difference equations that are calculated at every step $s_{1}(k d \tau)$. Writing $s_{1}(k d \tau)=\left(i+p^{k}\right) d x$ and using the first-order Euler approximation of $d s / d t$ we can obtain 


$$
p^{k+1}=p^{k}+\frac{d \tau}{d x^{2}}\left(\gamma_{s} \frac{\partial u_{s}}{\partial x}-\gamma_{l} \frac{\partial u_{l}}{\partial x}\right)
$$

This equation is valid for the solid-liquid moving boundary in Stages 2 and 3. For the liquid-vapor moving boundary the same Eq. (29), with $u_{l}$ in place of $u_{s}$, can be used with Eq. (17) to obtain the final form

$$
v^{k+1}=v^{k}+\frac{d \tau}{d x}\left(\gamma_{l} \frac{\partial u_{l}}{\partial x}+f(\tau)\right)
$$

where $v$ is the parameter used to track the movement of the boundary $s_{2}(t)=(i+v) d x$.

To manage the movement of the boundaries we update at every time step the fractional parameters $p$ and $v$. Their values lie between 0 and 1 , meaning the relative boundary lies between two nodes, except when $p>1$, which means that the moving boundary passed a node. To simulate this we remove the node from the solid domain and add a node to the liquid one. Calculating $p^{k+1}=p^{k}-1$ we obtain the starting $p$ for the spatial interval $d x$. For the liquid-vapor boundary we simply remove one node from the liquid domain without any additional operation, following the assumption that the vapor is continuously removed from the surface. This operation requires the determination of a temperature for the new node added in the liquid domain. Lacking physical principles to address this problem, (the conservation of energy has been already used for the Stefan equation and 
does not provide any further information) we can again use the Lagrangian interpolation of Eq. (26). Before adding the new node, with $p>1$, we can obtain the temperature in the position $x=(i+1) d x$ using the information at nodes $(i-1) d x$ (where $\left.u=u_{l}^{n_{l}-1}\right), i d x$ (where $\left.u=u_{l}^{n_{l}}\right)$ and $(i+p) d x$ (where $u=u_{B}$ by definition of $s_{1}$ ) obtaining:

$$
u_{l}^{i+1, k}=\frac{1-p}{1+p} u_{l}^{i-1, k}+\frac{2(p-1)}{p} u_{l}^{i, k}+\frac{2}{p(p+1)} u_{B}
$$

This is not a strict condition. The space and time steps are usually small, so it happens that $u_{l}^{i+1}$ is very close to $u_{B}$. We verified that imposing the condition $u_{l}^{i+1}=u_{B}$ does not change the result of the simulation if the total simulation time is not close to $\tau_{1}$.

\section{Thermal model validation}

To validate the code we used both an analytical solution and a test case found in the literature. Analytical solutions are very difficult to find and are only available for simple problems. For this reason we tested a simpler version of the code with only solid and liquid phases.

Analytical solution: the Neumann solution

The similarity solution originally obtained by Frank Neumann in 1860 for the solidification of a liquid phase can also be used for the inverse process. It is based on a particular solution of the heat equation that can be written in terms of error functions. 
The problem considered is the two phases one described by Eqs. (1), (2), (3) without taking into account the heat generation terms and with boundary and initial conditions

$$
\begin{aligned}
T_{l}=T_{0} & \text { for } \quad X=0, t \geqslant 0 \\
T_{s}=T_{i} & \text { for } \quad X=\infty, t \geqslant 0 \\
T_{l}=T_{s}=T_{m} & \text { for } \quad X=S_{1}(t), t \geqslant 0
\end{aligned}
$$

Following the known resolution described by Crank [2] with some variations, verified by comparison with Gupta [15] work, we can give the solution of (1) and (2) as

$$
\begin{gathered}
T_{l}=T_{0}+A \operatorname{erf}\left(\frac{X_{l}}{2 \sqrt{\alpha_{l} t}}\right) \\
T_{s}=T_{i}+B \operatorname{erfc}\left(\frac{X_{s}}{2 \sqrt{\alpha_{s} t}}\right)
\end{gathered}
$$

Let $S_{1}(t)$ be given by

$$
S_{1}(t)=2 \lambda \sqrt{\alpha_{l} t}
$$

where $\lambda$ is an unknown constant. For $X=S_{1}(t)$ Eqs. (38) and (39) are equal to $T_{m}$ for the boundary condition. Hence, with the previous definition 
of $S_{1}(t)$

$$
\begin{aligned}
& A=\frac{T_{m}-T_{0}}{\operatorname{erf}(\lambda)} \\
& B=\frac{T_{m}-T_{i}}{\operatorname{erfc}\left(\lambda \sqrt{\frac{\alpha_{l}}{\alpha_{s}}}\right)}
\end{aligned}
$$

Now, taking the derivatives of (38) and (39) and inserting into Eq. (3) with the obtained values of $A$ and $B$ we finally obtain

$$
\frac{\exp \left(-\lambda^{2}\right)}{\operatorname{erf}(\lambda)}+\frac{k_{s}}{k_{l}} \frac{\left(T_{m}-T_{i}\right)}{\left(T_{m}-T_{0}\right)} \sqrt{\frac{\alpha_{l}}{\alpha_{s}}} \frac{\exp \left(-\lambda^{2} \frac{\alpha_{l}}{\alpha_{s}}\right)}{\operatorname{erfc}\left(\lambda \sqrt{\frac{\alpha_{l}}{\alpha_{s}}}\right)}=-\frac{C_{m} \lambda \sqrt{\pi}}{c_{l}\left(T_{m}-T_{0}\right)}
$$

Calculating $\lambda$ from Eq. (40) with a numerical or graphical method we obtain the final time-dependent temperature profile and the movement of the boundary.

[Figure 4 about here.]

Fig. 4 shows the convergence of the numerical solution to the analytical one. Except at the very beginning of the simulation where it is not possible to start from zero for initialization issues, the difference between the two solutions quickly drops to less than $1 \%$ even with the biggest mesh size. This means that the accuracy of the calculation always grows in time, and the solution does not diverge. 
Comparison with previous numerical solution

Bonnerot et al. [5] proposed a solution for a two-phase Stefan problem using finite element method; they also modeled the appearance and disappearance of new phases. Our code has been modified to enforce an adiabatic condition at the end of the slab, as in the considered paper. The physical values used are:

$$
\begin{array}{ll}
T_{m}=1454 \mathrm{~K} & T_{v}=3000 \mathrm{~K} \\
T_{0}(X)=T_{0}=27 \mathrm{~K} & F(t)=F=2500 \mathrm{~W} / \mathrm{m}^{2} \\
c_{l}=c_{s}=1.7848 \mathrm{~J} /(\mathrm{kgK}) & \rho=2.77 \mathrm{~kg} / \mathrm{m}^{3} \\
a=1 \mathrm{~m} & k_{s}=0.259 \mathrm{~W} /(\mathrm{mK}) \\
k_{l}=0.259 \mathrm{~W} /(\mathrm{mK}) & C_{m}=779.8 \mathrm{~J} / \mathrm{kg} \\
C_{v}=13430 \mathrm{~J} / \mathrm{kg} &
\end{array}
$$

The results obtained for the boundary movement $S_{1}(t)$ and $S_{2}(t)$ are shown in Figures 5 and 6. The comparison with the code of Bonnerot et al. is shown in Figures 7 and 8 where the ratio between the values of the two solutions is plotted versus the time $t$. It is easy to observe that the two methods produced the same results, i.e., ratio close to 1 , with a difference smaller than $3 \%$, except where one of the two values is close to zero because of the numerical imposition of the boundary conditions ( slab thickness and axis origin). This pushes the ratio towards higher yet non physical values.

[Figure 5 about here.]

[Figure 6 about here.] 
[Figure 7 about here.]

[Figure 8 about here.]

Comparison with previous numerical solution

Another comparison with previous literature was carried out with the work of Belkin [16]. His experimental data can be normalized in order to obtain a self-similar curve of a dimensionless ejected mass independent of the heat flux incoming on the surface, while eliminating the dependence on the total time of the arc discharge. This allows us also to validate our model from the point of view of the vaporization process.

$$
\begin{aligned}
& M_{l}^{*}=\frac{M_{l}}{\frac{Q_{b}}{c_{s} T_{m}}} \\
& M_{v}^{*}=\frac{M_{v}}{\frac{Q_{b}}{c_{l} T_{m}}} \\
& {\left[q_{b} \sqrt{t_{d}}\right]^{*}=\frac{q_{b} \sqrt{t_{d}}}{T_{m} \sqrt{c_{l} \rho k_{l}}}}
\end{aligned}
$$

where $M_{l}^{*}$ and $M_{v}^{*}$ are the liquid and vapor nondimensional ejected mass, and $t_{d}$ is the total discharge time during which the flux $q_{b}$ is assumed constant.

[Figure 9 about here.]

Figures 9 shows the nondimensional mass flow rates for liquid and vapor phase, evaluated by our code, plotted versus a nondimensional parameter $\left[q_{b} \sqrt{t_{d}}\right]^{*}$ that is a function of the heat flux and the discharge time. The 
results are quite similar to those that can be found in [16]. Belkin identifies the onset of fusion as:

$$
\left[q_{b} \sqrt{t_{d}}\right]^{*}=\sqrt{\pi / 4}=0.885
$$

According to him, at low fluxes the metal is heated to fusion conditions at shallow depths and heat is transported deeper into the metal by thermal diffusivity. For high heat flux, i.e., large $\left[q_{b} \sqrt{t_{d}}\right]^{*}$, there is an intense vaporization of the surface and only a fraction of the energy is carried by conduction. Almost all the input energy is consumed by the vaporization of nearly all of the fused metal, leading to a small difference between $M_{l}^{*}$ and $M_{v}^{*}$. In our result, $\left[q_{b} \sqrt{t_{d}}\right]^{*}$ is about 0.7 , which is close to the Belkin's value.

It is interesting to note that the maximum of the $M_{l}^{*}$ curve, and so the onset of vaporization, can vary strongly with the imposed vapor temperature $T_{v}$. This means such a sort of self-similarity is not maintained if pressure varies during the experiment, because of the boiling point variation. Belkin developed an approximate expression for $M_{l}^{*}$ valid within the range $55 \cdot 10^{9}-$ $5 \cdot 10^{11} \mathrm{~W} / \mathrm{m}^{2}$

$$
M_{l_{\text {approx }}^{*}}^{*}=\frac{1.3}{\left[q_{b}{\sqrt{\tau_{d}}}^{*}\right.}\left[1-\frac{0.885}{\left[q_{b} \sqrt{\tau_{d}}\right]^{*}}\right]
$$

that, is only valid for one pressure. Nevertheless, some authors [13, 17] refer to this expression when calculating electrode evaporation without concern for this limitation. 


\section{Conclusions}

The numerical solution of a one dimensional, three-phases Stefan problem with a low Stefan number has been presented. The fundamental equations governing the phenomenon and the numerical approach used to solve the problem have been described. The results are in agreement with a previous solution obtained with a different numerical method and with experimental data found in literature. 


\section{Nomenclature}

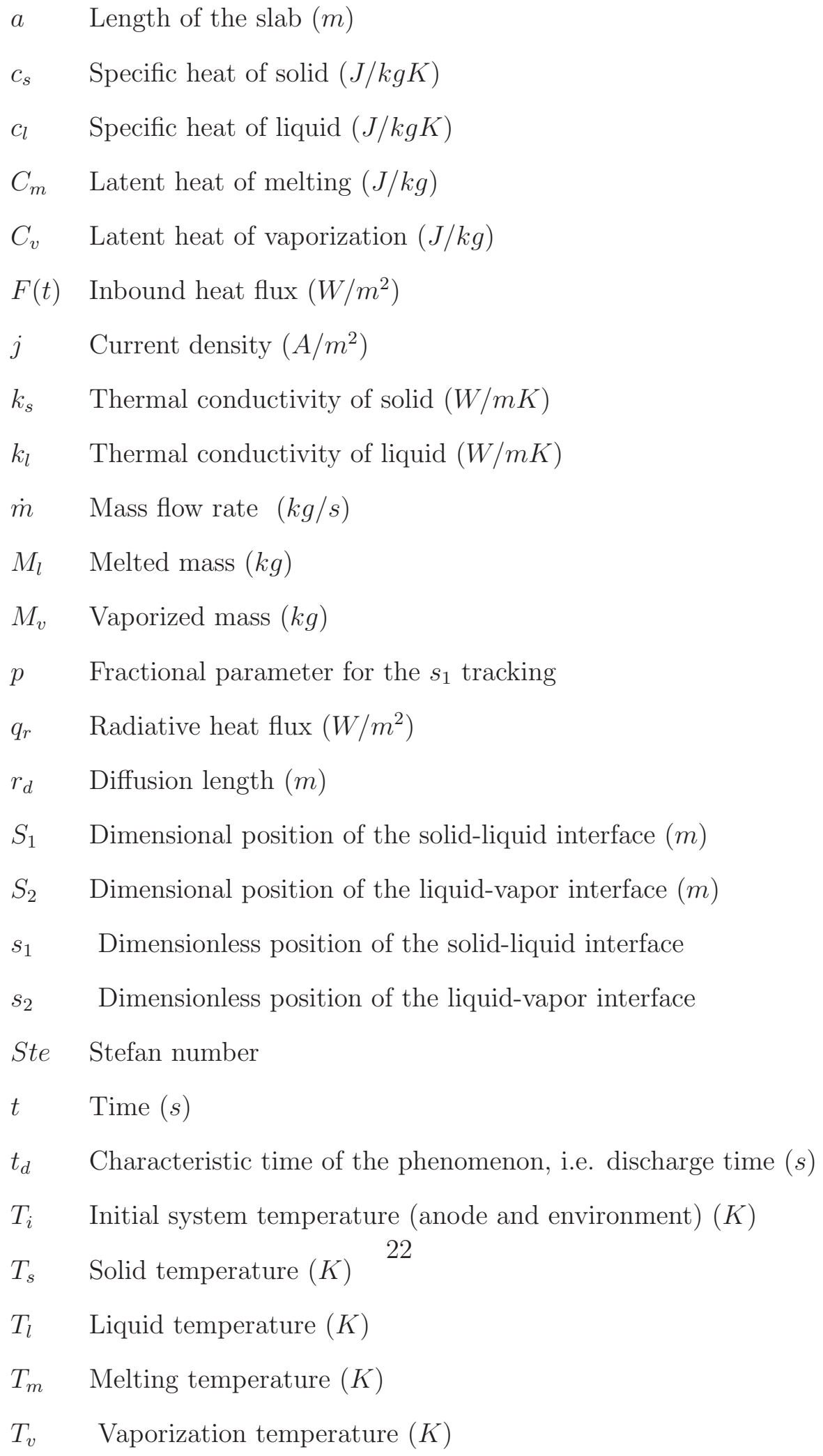


$u_{s} \quad$ Dimensionless solid temperature

$u_{l} \quad$ Dimensionless liquid temperature

$u_{m} \quad$ Dimensionless melting temperature

$u_{v} \quad$ Dimensionless vapor temperature

$v \quad$ Fractional parameter for the $s_{2}$ tracking

$X \quad$ Dimensional spatial coordinate $(m)$

$x \quad$ Dimensionless spatial coordinate

$X_{s}$ Dimensional spatial coordinate for the solid domain $(m)$

$x_{s} \quad$ Dimensionless spatial coordinate for the solid domain

$X_{l}$ Dimensional spatial coordinate for the liquid domain $(m)$

$x_{l} \quad$ Dimensionless spatial coordinate for the liquid domain

$\alpha_{s} \quad$ Diffusivity of the solid $\left.\left(\mathrm{m}^{2} / \mathrm{s}\right)\right)$

$\alpha_{l} \quad$ Diffusivity of the liquid $\left.\left(\mathrm{m}^{2} / \mathrm{s}\right)\right)$

$\eta_{s} \quad$ Resistivity of solid $(\Omega m)$

$\eta_{l} \quad$ Resistivity of liquid $(\Omega m)$

$\epsilon \quad$ Emissivity

$\rho \quad$ Density $\left(\mathrm{kg} / \mathrm{m}^{3}\right)$

$\tau \quad$ Dimensionless time 
[1] H. S. Carslaw, J. C. Jaeger, Conduction of heat in solids, Oxford University Press, $2^{\text {nd }}$ edition edition, 1959.

[2] J. Crank, Free and moving boundary problems, Oxford University Press, 1984.

[3] A. Kharab, Computer Methods in Applied Mechanics and Engineering 145 (1997) 217-225.

[4] D. R. Atthey, IMA Journal of Applied Mathematics 13 (1974) 353-366.

[5] R. Bonnerot, P. Jamet, Journal of Computational Physics 41 (1981) 357-388.

[6] E. Javierre, C. Vuik, F. Vermolen, A. Segal, Journal of Computational Physics 224 (2007) 222-240.

[7] M. Muradoglu, G. Tryggvason, Journal of Computational Physics 227 (2008) 2238-2262.

[8] S. Pang, L. Chen, J. Zhou, Y. Yin, T. Chen, Journal of Physics D: Applied Physics 44 (2011) 025301 (15 pp).

[9] W. R. B. J. M. Somerville, N. H. Fletcher, Proceedings of the Physical Society 65 (1952) 963-970.

[10] J. M. Somerville, C. T. Grainger, British Journal of Applied Physics 7 (1956) 109-110. 
[11] J. M. Somerville, J. F. Williams, Proceedings of the Physical Society 74 (1959) 309-315.

[12] I. I. Beilis, IEEE Transactions on Plasma Science 29 (2001) 657-670.

[13] F. M. Lehr, M. Kristiansen, IEEE Transactions on Plasma Science 17 (1989) 811-817.

[14] M. N. Özisik, Heat conduction, John Wiley and Sons, 1980.

[15] S. C. Gupta, The classical Stefan problem. Basic concepts, modelling and analysis, North Holland Series in Applied Mathematics and Mechanics, Elsevier, 2003.

[16] G. S. Belkin, Soviet Physics - Technical Physics 15 (1971) 1167-1170.

[17] P. Borkowsky, M.Hasegawa, IEEE Transactions on Electron Devices E90-C (2007) 1369-1376. 


\section{List of Figures}

1 Spots on aluminum anode . . . . . . . . . . . . . 27

2 Stages of the model . . . . . . . . . . . . . . . . . 28

3 Lagrangian type interpolation for solid-liquid boundary . . . . 29

4 Percentual error of our calculated $S_{1}(t)$ with respect to Neumann's analytical solution. Error is provided for different mesh sizes: the legend shows the mesh size with respect to the total length of the slab. . . . . . . . . . . 30

5 Our code simulation of Bonnerot's numerical problem: showing appearance, movement and disappearance of the free boundaries on the $x-t$ plane. Solid, liquid and vapor phase appear at incresing $t$. Heat flux is applied at $x=1 . \ldots . . . . .31$

6 Our code simulation of Bonnerot's numerical problem: velocities of the free boundaries $d S_{1} / d t$ and $d S_{2} / d t$ plotted Vs. time. 32

7 Comparison between our code and Bonnerot's code. Ratios of the boundary positions $S_{1}$ (continuous line) and $S_{2}$ (dashed line) with respect to time calculated with our code and Bonnerot's code. . . . . . . . . . . . . . . 33

8 Comparison between our code and Bonnerot's code. Ratios of the velocities of the free boundaries $d S_{1} / d t$ (continuous line) and $d S_{2} / d t$ (dashed line) with respect to time calculated with our code and Bonnerot's code. . . . . . . . . . . . . . . 34

9 Results of our code. Normalization of copper simulation results according to Belkin method for evaluating experimental data. . . . . . . . . . . . . . . . . . 35 


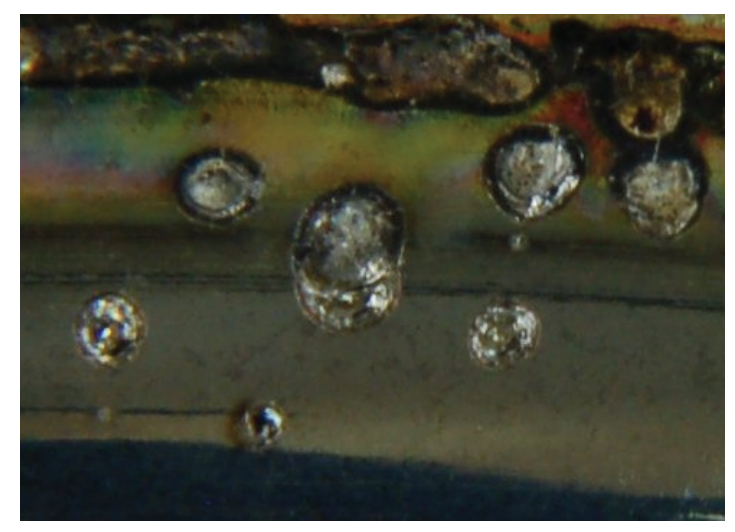

Figure 1: Spots on aluminum anode 


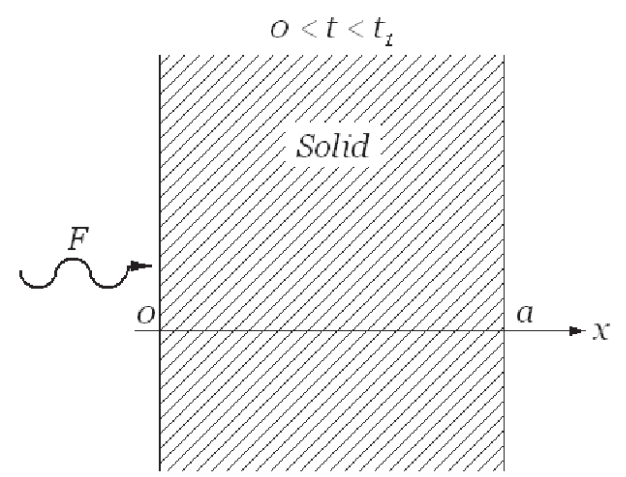

Stage 1

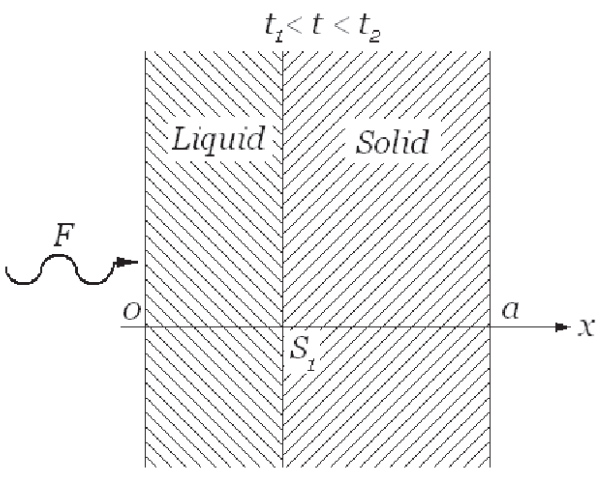

Stage 2

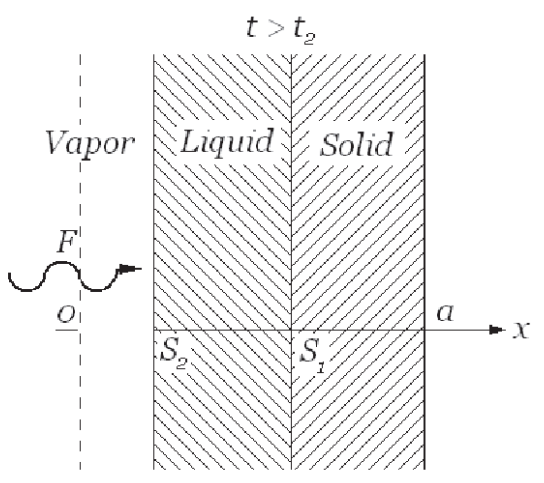

Stage 3

Figure 2: Stages of the model 


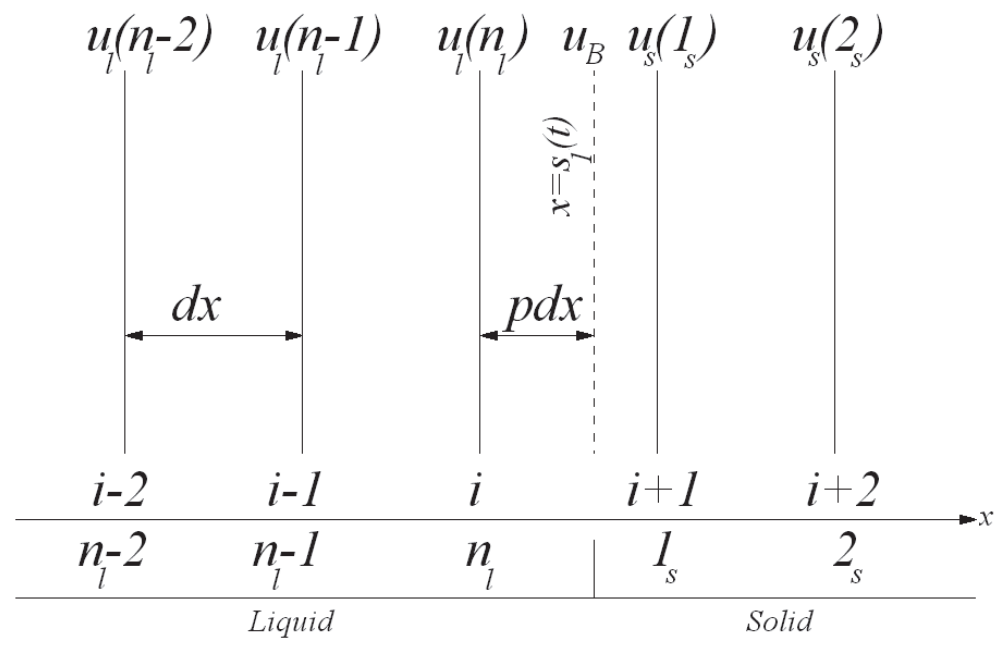

Figure 3: Lagrangian type interpolation for solid-liquid boundary 


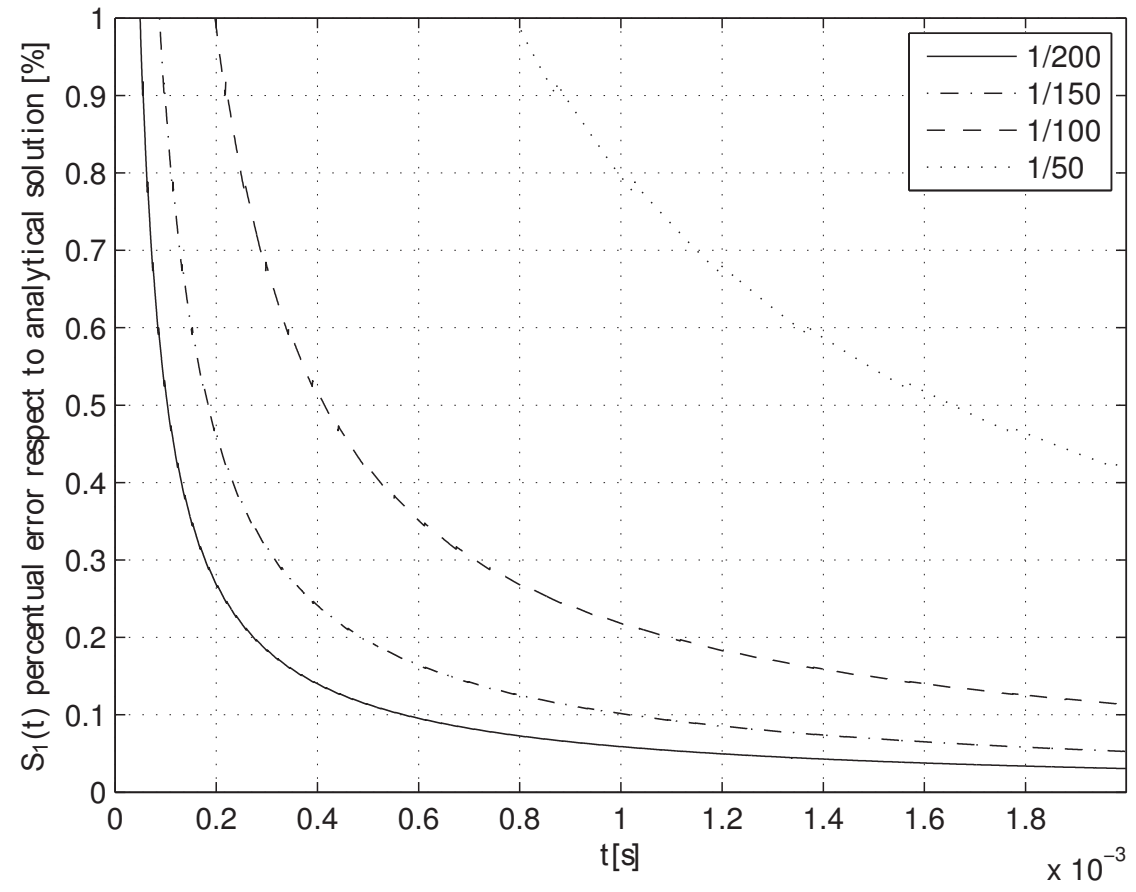

Figure 4: Percentual error of our calculated $S_{1}(t)$ with respect to Neumann's analytical solution. Error is provided for different mesh sizes: the legend shows the mesh size with respect to the total length of the slab. 


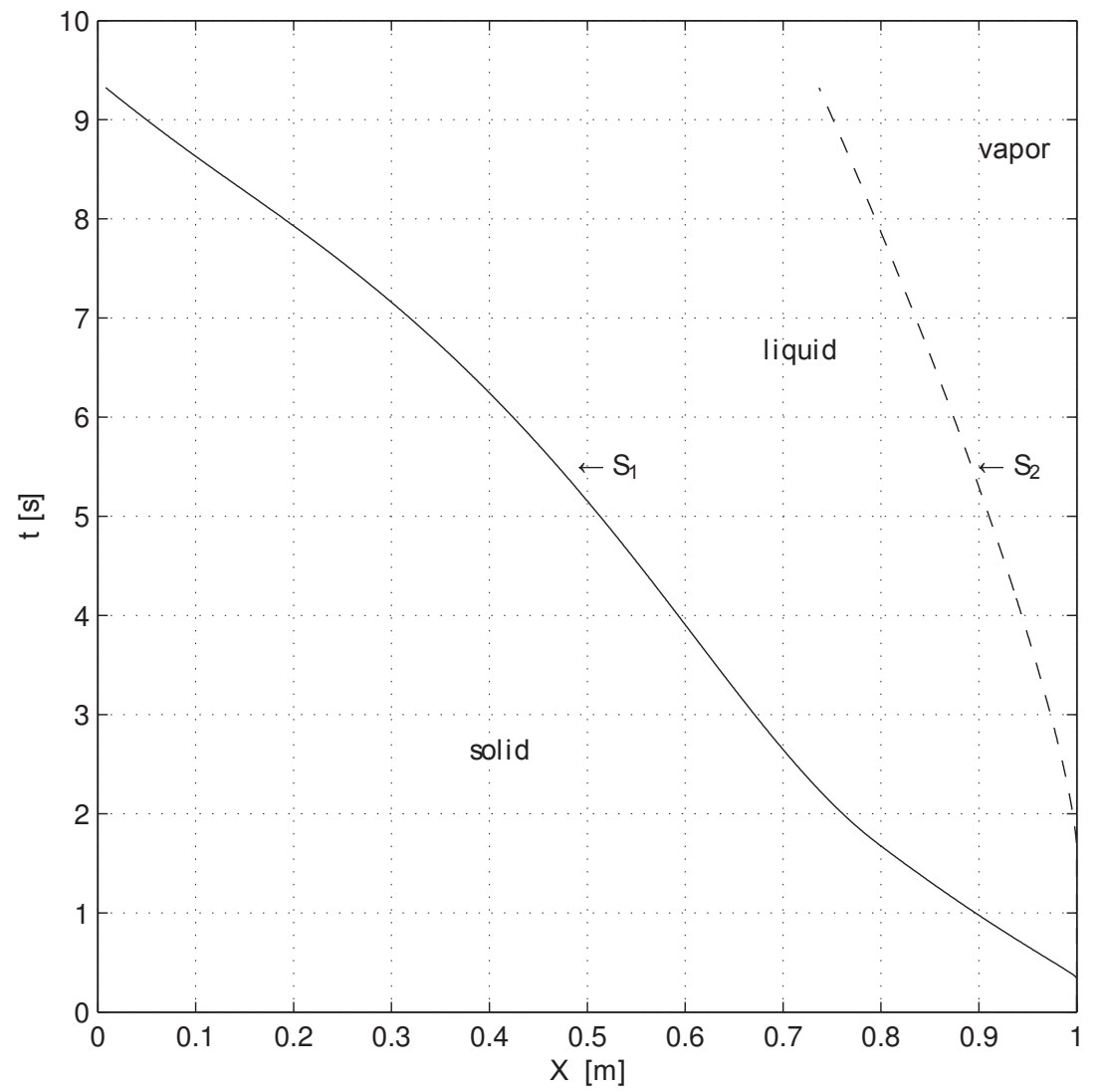

Figure 5: Our code simulation of Bonnerot's numerical problem: showing appearance, movement and disappearance of the free boundaries on the $x-t$ plane. Solid, liquid and vapor phase appear at incresing $t$. Heat flux is applied at $x=1$. 


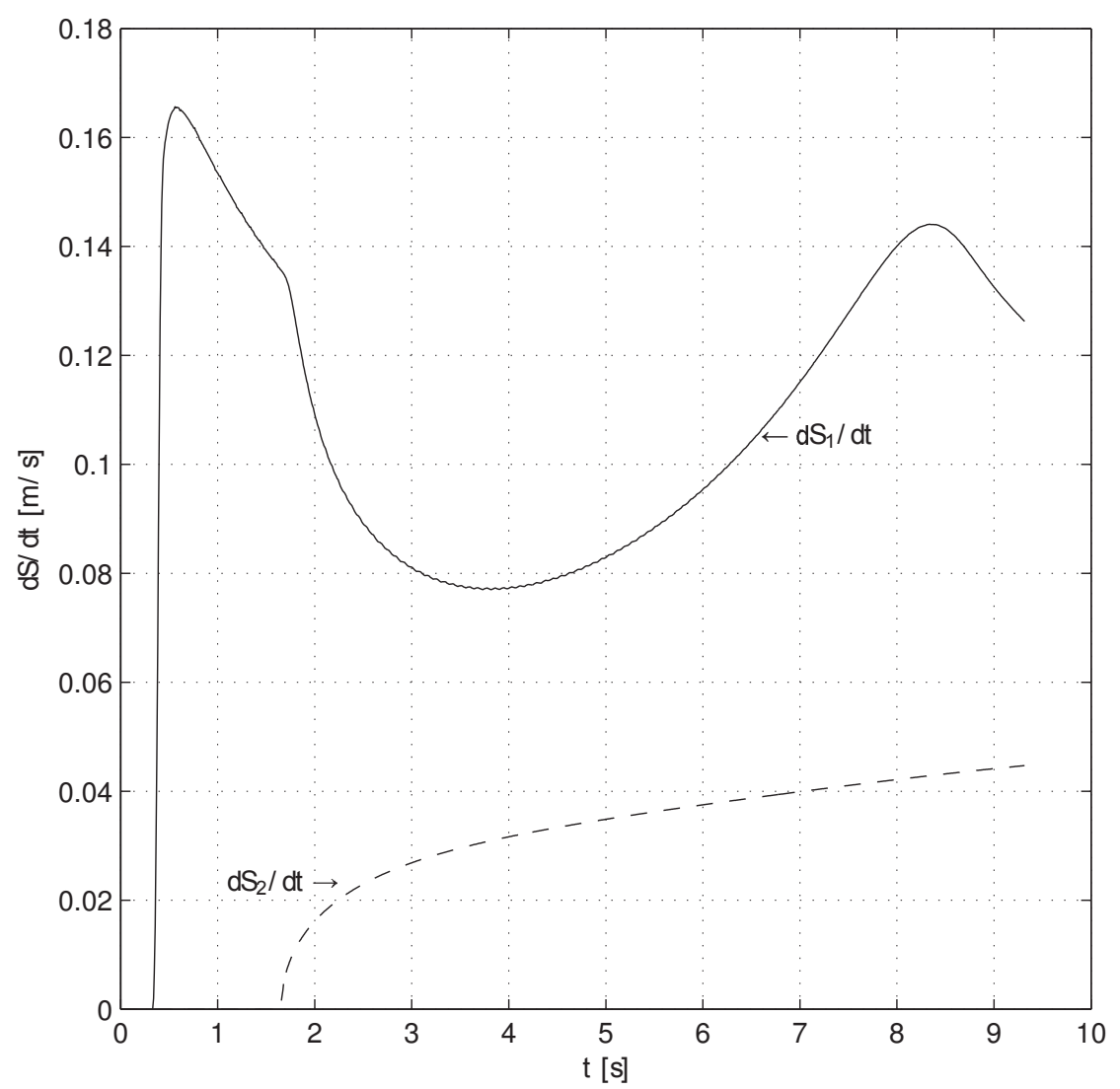

Figure 6: Our code simulation of Bonnerot's numerical problem: velocities of the free boundaries $d S_{1} / d t$ and $d S_{2} / d t$ plotted Vs. time. 


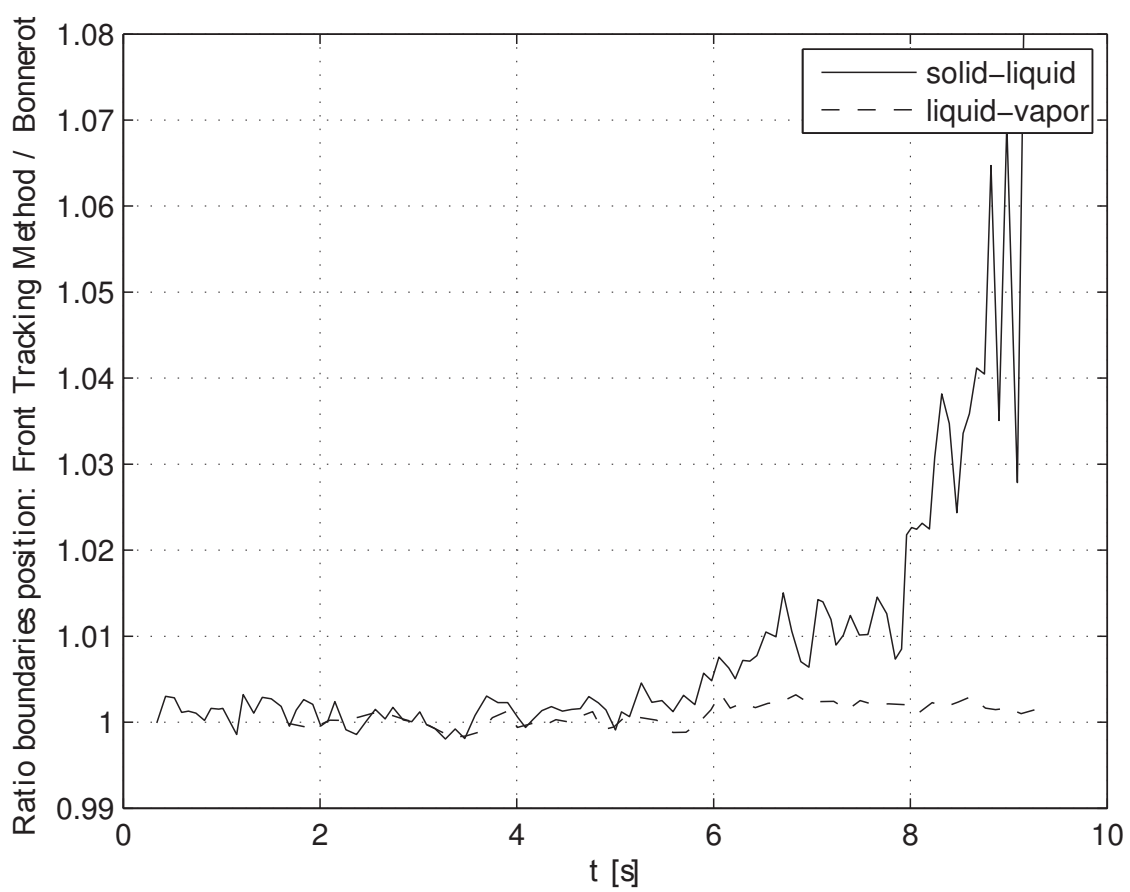

Figure 7: Comparison between our code and Bonnerot's code. Ratios of the boundary positions $S_{1}$ (continuous line) and $S_{2}$ (dashed line) with respect to time calculated with our code and Bonnerot's code. 


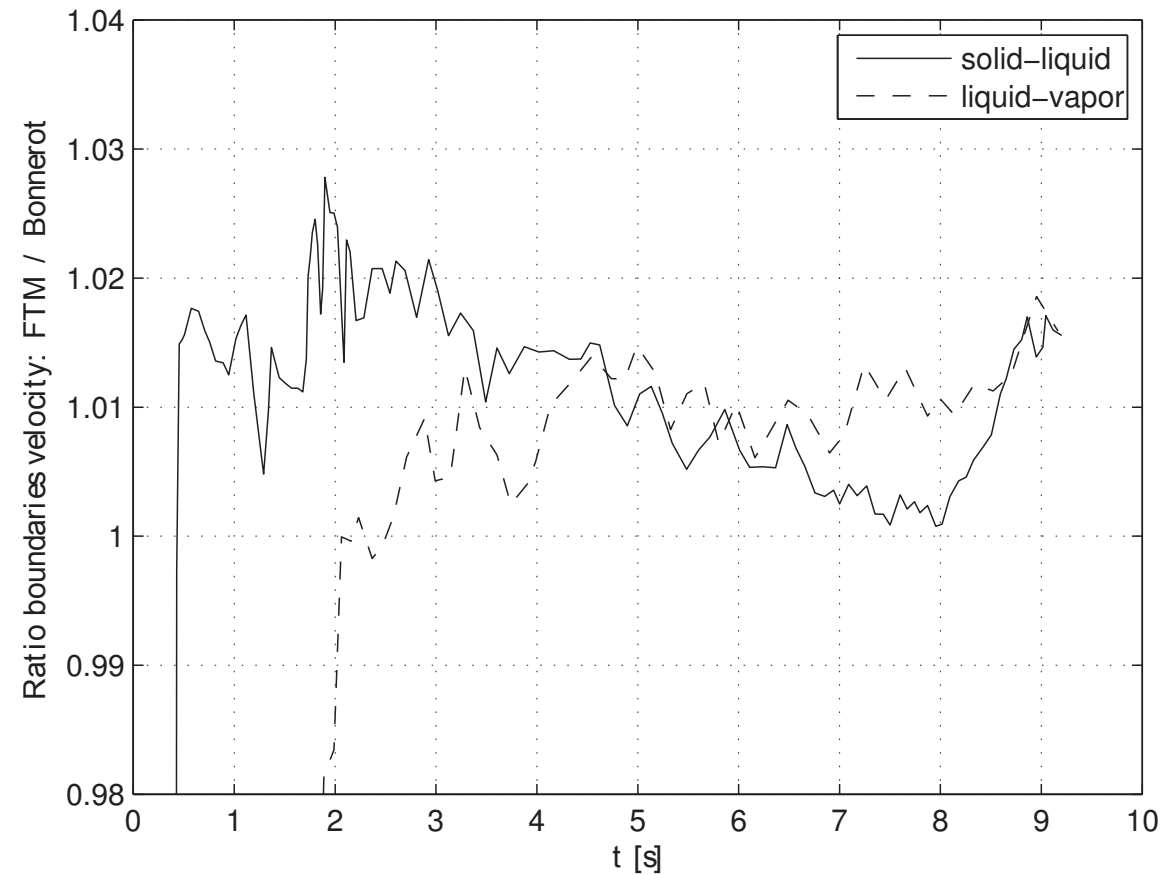

Figure 8: Comparison between our code and Bonnerot's code. Ratios of the velocities of the free boundaries $d S_{1} / d t$ (continuous line) and $d S_{2} / d t$ (dashed line) with respect to time calculated with our code and Bonnerot's code. 


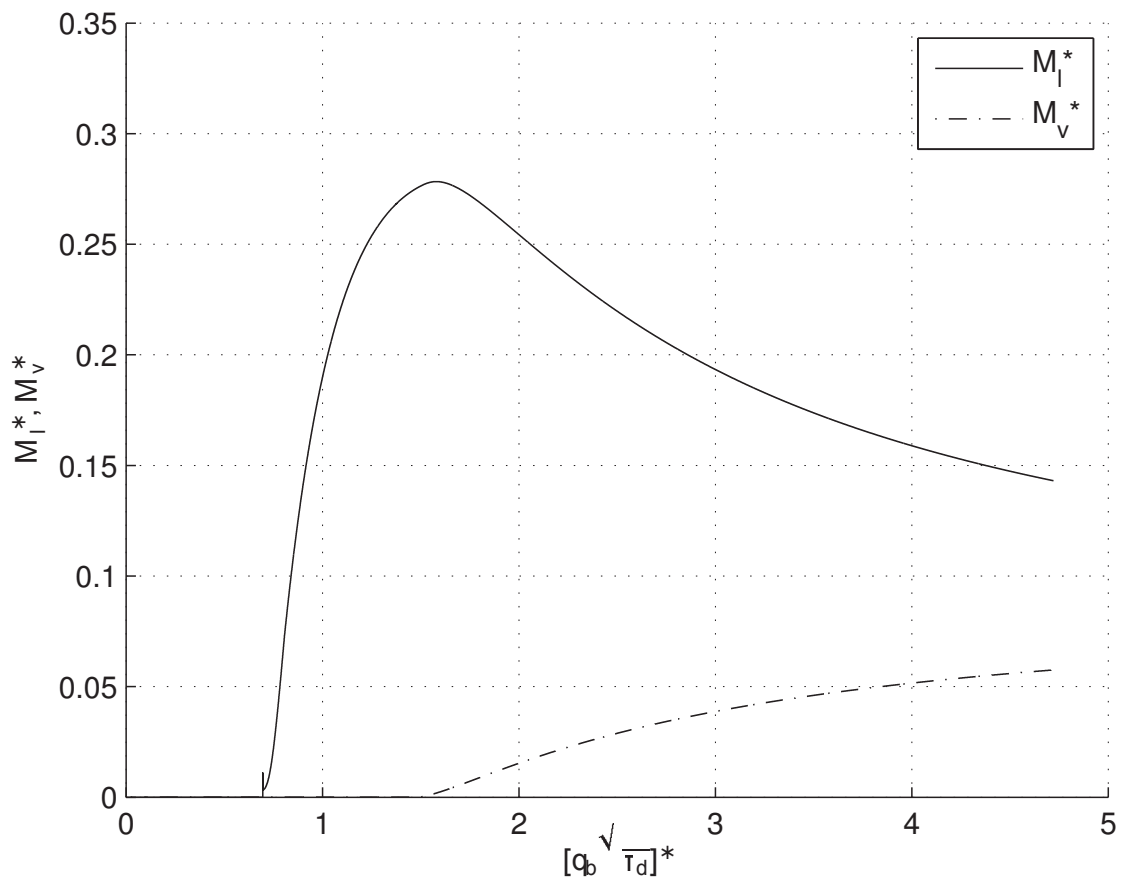

Figure 9: Results of our code. Normalization of copper simulation results according to Belkin method for evaluating experimental data. 